\title{
Zur Ratifizierungskrise des Verfassungsvertrages - drei politikwissenschaftliche Lesarten und ihre Kritik
}

\author{
Brigid Laffan und Imogen Sudbury*
}

Was ist die Natur der politischen Ordnung der EU? Angesichts der großen Uneinigkeit über diese Frage ist der europäische Integrationsprozess einem pragmatischen, stück- und schrittweisen Entwicklungspfad gefolgt, der sorgfältig gestaltet wurde, um konkurrierende Visionen der EU oder dessen, was sie sein sollte, mit zu umfassen. Diese „konstruktive Ambiguität ${ }^{\text {1 }}$ im Herzen des Integrationsprozesses hat zu einem ungeheuer komplexen, differenzierten und multi-dimensionalen System geführt, das sich den traditionellen Kategorisierungen der politischen Theorie entzieht. Die Analyse dieses Systems verlangt ein hohes Maß an Kreativität von Politikwissenschaftlerinnen und Politikwissenschaftlern, die nicht nur hinsichtlich der konstitutionellen Ausgestaltung der EU gespalten sind, sondern sich auch über die Quellen der Legitimität der EU, die Natur ihrer Integrationsdynamiken und die Auswirkungen der EU auf ihre Mitgliedstaaten uneinig sind. Europäische Integration ist sowohl im Bereich der Politik als auch unter Wissenschaftlerinnen und Wissenschaftlern ein strittiges Projekt.

Der Verfassungsprozess war ein weiterer Versuch, diese politische Ordnung zu definieren und zu gestalten. Ob die endgültige Fassung des Verfassungsvertrages den Zielen der Reformer entsprach oder nicht, so schrieben doch die Kommentare, die den Prozess begleiteten, dem Vertrag nicht weniger als eine unerlässliche Rolle bei der Sicherstellung von Effizienz und Demokratie in einem Europa der 25 zu. Romano Prodi zum Beispiel beschrieb den Prozess als ein „grand project of creating European Union“, das erforderlich sei „to take the European project forward“. ${ }^{2}$ Es überrascht daher nicht, dass das Scheitern des Verfassungsvertrages imstande gewesen ist, eine so intensive Debatte über die Implikationen für die Zukunft der EU auszulösen.

Diese Debatte ist von großem Interesse für das EU-Exzellenznetzwerk ,EU-CONSENT“, das von der Beobachtung ausgeht, dass die Erweiterungsrunde 2004 zum ersten Mal die Frage aufwarf, ob Vertiefung und Erweiterung in Zukunft zwei einanderentgegen gesetzte Pole darstellen könnten. Das Netzwerk zielt auf die Untersuchung der Beziehung zwischen diesen beiden Kräften, die bis heute als sich wechselseitig verstärkende Integrationsmechanismen genutzt worden sind, sowie auf die Prüfung von Szenarien für die Zukunft der EU. Was verrät uns das Scheitern der Ratifikation des Verfassungsvertrages demnach über die Natur der Europäischen Union, wie sie sich gegenwärtig darstellt, und was über ihren wahrscheinlichen Entwicklungspfad? Impliziert das Scheitern der Ratifikation, dass Erweiterung und Vertiefung eventuell nicht länger Hand in Hand gehen? Sind weitere Integrationsschritte jetzt möglich, und können weitere Erweiterungen dennoch vollzogen werden? Ist die

* Prof. Dr. Brigid Laffan, Direktorin, Dublin European Institute; Jean-Monnet-Professorin, University College Dublin.

Imogen Sudbury, M.A., wissenschaftliche Mitarbeiterin, Dublin European Institute, University College Dublin.

1 Anne Faber/Wolfgang Wessels: Wider Europe, Deeper Integration? Constructing Europe Network, Background Paper, EU-CONSENT Network of Excellence, abrufbar unter: http://www.eu-consent.net/content.asp?contentid=740 (letzter Zugriff: 10.10 .206 ).

2 Romano Prodi zitiert in: Johan P. Olsen: Reforming European Institutions of Governance, in: Journal of Common Market Studies 4/2002, S. 581-602. 
EU zu einer politischen Ordnung geworden, die sich in einem Gleichgewichtszustand eingependelt hat? Oder ist möglicherweise ein Scheitern bei dem Versuch, weiter voran in Richtung einer vertieften Integration zu schreiten, an sich bereits ein Indikator für Desintegration?

Dieser Beitrag nimmt die Debatte über die Ratifizierungskrise als Linse, durch die unterschiedliche theoretische Standpunkte zur Natur der politischen Ordnung der EU nach der Erweiterung von 2004 betrachtet werden. Der erste Abschnitt prüft, wie Wissenschaftlerinnen und Wissenschaftler aus drei unterschiedlichen theoretischen Traditionen die Verfassungskrise interpretiert haben. Obwohl es allgemeine Übereinstimmung darüber gibt, dass die Situation keine Katastrophe für die Zukunft des Integrationsprozesses ist, werden ihre Argumente durch drei sehr unterschiedliche Beschreibungen der EU, drei unterschiedliche ,Rezepte ' für die weitere ,Gesundheit' der EU und drei unterschiedliche Prognosen der zukünftigen Entwicklung der EU untermauert. Der zweite Abschnitt diskutiert jeden dieser drei Standpunkte kritisch und unter Einbeziehung der theoretischen Konzepte, die von Stein Rokkan in seiner Untersuchung der politischen Systemgestaltung entwickelt wurden. Eine Analyse aus dieser Perspektive zeichnet ein ambivalenteres Bild von der Zukunft der EU und legt den Schluss nahe, dass die Ambiguität, die auf die gegenwärtige „lose begrenzte“ Natur der EU zurückzuführen ist, unterschwellige Spannungen erzeugt, die letzten Endes zu ihrer Desintegration führen könnten.

\section{Drei Lesarten der EU als politische Ordnung in der Ratifizierungskrise}

\section{Die EU als Problemlösungsgemeinschaft}

Gegen die Vielzahl von Stimmen, die nach Mitteln suchen, um Regieren auf supranationaler Ebene zu legitimieren, haben Giandomenico Majone und Andrew Moravcsik argumentiert, dass der Mangel an Bürgerbeteiligung und Politisierung ein Imperativ für, und nicht eine Sperre gegen legitimes europäisches Regieren sei. Diese beiden Wissenschaftler haben eine normative Sicht der EU als einer Problemlösungsgemeinschaft vorgestellt, deren Ziel es ist, einvernehmliche Lösungen für gemeinsame Probleme zu erzielen, welche nicht von den Nationalstaaten im Alleingang gelöst werden können. Der Entwicklungspfad der EU wird jedoch von der nationalen politischen Führung kontrolliert.

In einer Reihe von Artikeln hat Moravcsik das Scheitern des Verfassungsvertrages herangezogen, um die Forderungen derjenigen zu widerlegen, die behaupten, dass die EU demokratisiert werden müsse, um ein als solches wahrgenommenes demokratisches Defizit zu bekämpfen. Er zieht zwei zentrale Lehren aus dem Scheitern der Ratifizierung. Die erste ist, dass die EU als politisches System einen stabilen Gleichgewichtszustand sowohl in institutioneller wie auch in materieller Hinsicht erreicht hat. Die zweite ist, dass dieses System sowohl normativ wünschenswert als auch vollständig legitim ist. ${ }^{3}$

Moravcsiks Ausgangspunkt ist, dass das Scheitern des Verfassungsvertrages keine Krise für die EU darstellt, weil bereits eine ,konstitutionelle Ordnung ' in der Europäischen Union besteht. Auf materieller Ebene ist eine Kompetenzaufteilung zwischen der EU und den Mitgliedstaaten beschlossen worden, und die Balance hat sich stabilisiert. „The EU is now preeminent in trade, agriculture, fishing, Eurozone monetary policy and some business regulation and helps coordinate cooperation in foreign policy. “4 Laut Moravcsik macht dieser le-

3 Andrew Moravcsik: What Can We Learn from the Collapse of the European Constitutional Project?, in: Politische Vierteljahresschrift 2/2006, S. 219-241.

4 Andrew Moravcsik: Europe without Illusions, in: Prospect 112, Juli 2005, S. 4. 
gislative Output wenig mehr als 20 Prozent aller Gesetzgebung aus und korrespondiert mehr oder weniger mit den Bereichen, in denen die Bürgerinnen und Bürger sich explizit ein Handeln der EU wünschen. Sie stellen ,,areas of less concern, or on which there is an administrative, technical or legal consensus" ${ }^{\circ 5}$ dar.

Moravcsik argumentiert, dass diese Bereiche genau diejenigen sind, in denen sogar auf der Nationalstaatsebene entpolitisiertes Regieren als normativ legitim angesehen wird. ${ }^{6}$ Diese Sichtweise wird von Majone geteilt, dessen wohlbekannte These, dass die EU ein ,regulativer Staat" sei, von der Prämisse ausgeht, dass es das Ziel der EU sei, regulative Fragen zu regeln, die pareto-effiziente ${ }^{7}$ oder Win-win-Lösungen erfordern. Die europäischen Regierungen haben diese Kompetenzen genau aus dem Grund an die supranationale Ebene delegiert, um sie von politischer Einmischung abzuschirmen, die die Pareto-Effizienz durch die Schaffung eines Potenzials für eine politische ,Inbesitznahme " dieser Fragen durch mächtige Minderheiten oder kurzfristige politische Handlungserfordernisse bedrohen würde. ${ }^{8}$ Pettit entwickelt dieses Argument weiter: „Democracy requires modes of popular and political control, of course, but it also requires a regime under which people and politicians are willing and able to trust in various depoliticised bodies to make decisions on certain matters of common interest. The democratic society which leaves the exercise of power to popular majorities and political elites may easily become the worst of despotisms. "9 Moravcsik erhält das Argument aufrecht, dass es keinen politischen Willen gebe, den Integrationsprozess weiter zu vertiefen, da die Mitgliedstaaten bereits alle Kompetenzen delegiert hätten, die solche „matters of common interest“ darstellen. Als Beleg dieses Arguments stellt er fest, dass der Verfassungsvertrag - anders als die Einheitliche Europäische Akte (EEA), der Vertrag über die Europäischen Union (Maastricht) oder der Vertrag von Amsterdam - durch seinen verblüffenden Mangel an positiven Reformvorschlägen auffällt. ${ }^{10}$ Da supranationale Institutionen nur den Willen der Mitgliedstaaten ausführten und die Mitgliedstaaten kein Interesse an einer Vertiefung der Integration zeigten, sei eine konstitutionelle Einigung bezüglich der Substanz europäischer Zusammenarbeit erzielt worden.

Wenn man dieses Argument akzeptiert, folgt daraus, dass die Legitimität europäischen Regierens davon abhängen wird, ob ihr institutionelles Design sich als effizient erweist bei der Erreichung wünschenswerter Ergebnisse, die sowohl transparent sind als auch einer politischen Verantwortung zugeordnet werden können. Laut Moravcsik ist in der Tat eine institutionelle Dimension der konstitutionellen Einigung erreicht worden, die diese Bedingungen erfüllt. Delegierte Politiken seien einem empfindlichen und komplexen System der Gewaltenteilung und -verschränkung unterworfen, das sicherstelle, dass nur die Politiken durchsetzbar seien, bei denen ein großes gegenseitiges Einvernehmen besteht. Die Mitgliedstaaten behielten die Kontrolle über die Richtung der Integration und verhandelten die wichtigsten Entscheidungen in hartem, intergouvernementalen Bargaining. Alle Politikvorschläge, die von der Kommission vorgelegt werden, seien das Ergebnis einer Konsultation breiter gesellschaftlicher Interessen, und um angenommen zu werden, müsse ihnen dann entweder der Ministerrat einstimmig zustimmen, oder aber sie müssten - in der Mehrzahl der Fälle - hohe

5 Moravcsik: Europe without Illusions, S. 8.

6 Moravcsik: What Can We Learn from the Collapse of the European Constitutional Project?

7 Das Pareto-Optimum bezeichnet einen Zustand, in dem kein Individuum besser gestellt werden kann, ohne ein anderes dadurch schlechter zu stellen.

8 Giandomenico Majone: The Rise of the Regulatory State in Europe, in: West European Politics 3/1994, S. 77101.

9 Philip Pettit: Deliberative Democracy And The Case For Depoliticising Government, 2001, abrufbar unter: http://www.austlii.edu.au/au/journals/UNSWLJ/2001/58.html\#Heading125 (letzter Zugriff: 10.10.2006).

10 Moravcsik: Europe without Illusions, S. 3. 
Mehrheitsquoren sowohl im Ministerrat als auch im Europäischen Parlament erfüllen. Die nationalen Parlamente und das Europäische Parlament üben jeweils Kontrollfunktionen aus, und der Europäische Gerichtshof übt die richterliche Überprüfung aller Handlungen der EU aus. ${ }^{11}$ Kurzum: ,,[O]nce we set aside ideal notions of democracy and look to real world standards, we see that the EU is at least as transparent, responsive accountable and honest as its member states." 12

Dementsprechend erhält Moravcsik das Argument aufrecht, dass der Verfassungsvertrag nicht wegen seines Inhaltes, einem ,,conservative text containing incremental improvements which consolidate EU developments of the past 20 years" "13, sondern wegen des Diskurses gescheitert sei, von dem die politischen Eliten Gebrauch gemacht hätten, um ihn zu, verkaufen'. Dieser Diskurs habe die Basis der bestehenden konstitutionellen Einigung unterminiert. Der Verfassungsvertrag wurde regelmäßig als ,,a grand scheme for constitutional revision and popular democratization of the EU"14 bezeichnet. Moravcsik sieht im Scheitern des Verfassungsvertrages den endgültigen Beweis dafür, dass diese Art von Demokratisierung der EU nicht angemessen ist. Er stellt insbesondere die vorgefasste Logik in Frage, dass Beteiligungsmöglichkeiten eine informierte Deliberation erzeugen, die ihrerseits Identität, Vertrauen und Legitimität generieren. Er sieht den Grund für das geringe Engagement der Bürgerinnen und Bürger in EU-Fragen darin, dass die Union mit Fragen von geringer politischer Bedeutung befasst sei und dass die Beteiligungskosten in Form von Lernprozessen, Deliberation und Mobilisierung schlicht zu hoch seien, als dass rationale Individuen sie in Betracht zögen: ,[S]ince everyday voters view the matters handled by the EU as relatively obscure, they have little incentive to debate or decide them. " ${ }^{15}$ Darüber hinaus erfordere politische Deliberation eine politische Strukturierung wie politische Konfliktlinien, intermediäre Organisationen und kulturelle Bindungen. Eine Politisierung auf der EU-Ebene, die diese Bedingungen nicht erfülle, öffne die Debatte für extreme Sichtweisen der EU auf beiden Seiten des politischen Spektrums. Daher gebe es wenig Grund zu glauben, dass die Bürgerinnen und Bürger, denen EU-Fragen vorgelegt würden, eine gut informierte und überlegte Wahl treffen werden. ${ }^{16}$ Dies erklärt, warum die Debatten um die Referenden durch innenpolitische Belange dominiert wurden und warum am Ende des Verfassungsprozesses wenige darstellen konnten, was der Inhalt des entstandenen Dokumentes ist. ${ }^{17}$

Für Moravcsik bestätigte das Scheitern des Verfassungsvertrages und der Referenden daher eine Beschreibung der EU als einer Problemlösungsgemeinschaft, an die die Mitgliedstaaten rational diejenigen Bereiche delegiert haben, die am effektivsten auf der supranationalen Ebene behandelt werden können. Dieses System funktioniert auf der Basis einer konstitutionellen Einigung, deren Legitimität sich aus indirekten Formen der demokratischen Kontrolle und einem stabilen institutionellen Arrangement der Gewaltenteilung und verschränkung ableitet. Die Existenz der EU lässt den Mitgliedstaaten die volle Kontrolle über die Funktionen, die auf der nationalen Ebene verbleiben - wie die Wohlfahrtssysteme, die einer partizipatorischen Demokratie unterworfen sind. Moravcsiks ,Rezept ${ }^{\star}$ ist, dass nicht mehr als ein ,Basteln“ an institutionellen Designs vonnöten sei, um eine ,gesunde“ Fortführung dieses Systems zu gewährleisten. Im Gegensatz dazu würde demokratische Par-

11 Moravcsik: What Can We Learn from the Collapse of the European Constitutional Project?

12 Moravcsik: Europe without Illusions, S. 8.

13 Moravcsik: Europe without Illusions, S. 3.

14 Moravcsik: Europe without Illusions, S. 2.

15 Moravcsik: What Can We Learn from the Collapse of the European Constitutional Project?, S. 226.

16 Moravcsik: What Can We Learn from the Collapse of the European Constitutional Project?, S. 227.

17 Moravcsik: What Can We Learn from the Collapse of the European Constitutional Project?, S. 228. 
tizipation die Effizienz und Stabilität dieses Systems in Gefahr bringen. Obwohl er keine Vorhersage für die Zukunft der EU trifft, folgt aus seiner Argumentation, dass eine weitere Vertiefung nicht zu erwarten ist, dass aber eine weitere Erweiterung möglich ist, wenn sie den Interessen der Mitgliedstaaten gerecht und als kompatibel mit der konstitutionellen Konfiguration erachtet wird. Kurzum, die EU sei jetzt ein ,reifes' politisches System und „,does not need to move forward on a neo-functionalist bicycle in order to be stable." 18

\section{Die EU als ein demokratisches politisches Gemeinwesen}

Hix teilt Moravcsiks Ansicht, dass das Scheitern der Ratifikation keine Krise für die EU darstellt, und er stimmt darin überein, dass die EU im Hinblick auf ihre institutionelle und substanzielle Entwicklung einen Gleichgewichtszustand erreicht hat. Die ökonomische und politische Architektur sei weitgehend vollständig und umfasse ,a continental scale market regulated at the European level, taxing and spending at the national level and the coordination of national policies where one member state"s policies affect another." 19

Für Hix deutet eine Bewertung der Ratifikationskrise jedoch auf die Tatsache hin, dass die Legitimität der EU in der Bevölkerung dringend gestärkt werden muss, um langfristige Effektivität und Stabilität zu gewährleisten. Zusammen mit anderen Wissenschaftlern, die Moravcsik für seinen übertrieben optimistischen Glauben verspotten, dass die EU die beste aller möglichen Welten ${ }^{20}$ biete, argumentiert Hix, dass eine stärkere Demokratisierung der europäischen Politikgestaltung angesichts der heutigen Natur und Reichweite der EU als einem politischen System sowohl unvermeidlich als auch wünschenswert sei. ${ }^{21}$

Hix geht von der Prämisse aus, dass wenige EU-Politiken tatsächlich die Art von paretoeffizienten Ergebnissen produzieren, auf denen Moravcsik und Majone ihre Argumentation aufbauen. Vielmehr produziere der europäische Entscheidungsfindungsprozess Gewinner und Verlierer. Sogar im Bereich der Regulierung des Binnenmarktes, behauptet Hix, seien die Entscheidungen, die getroffen werden, politischer Natur und hätten bedeutende redistributive Konsequenzen. So habe zum Beispiel die Liberalisierung durch die Beseitigung nationaler Protektion und Handelsbarrieren negative Konsequenzen für einheimische Produzenten gehabt. ${ }^{22}$ Vertreter der politischen Ökonomie stimmen dem zu: „What kind of minimum common standards or policies we want to adopt at the EU level for a wide range of policy areas related to the functioning of the internal market is a real and, indeed, legitimate political issue. It is not just a matter for technocrats to resolve." 23

Ferner weist Hix darauf hin, dass die heutige EU nicht nur Entscheidungen über Regulierungsmaßnahmen trifft, sondern über ein ganzes Spektrum von unterschiedlichen Politiken - von Fragen, die das Gemeinwohl berühren, bis hin zu reinen Umverteilungsfragen. Nachdem die grundlegende konstitutionelle Architektur nun errichtet worden sei, müssten schwierige ideologische Entscheidungen über den Einsatz dieses politischen Apparats getroffen werden: „Should the EU single market be more liberal or regulated? Should macro-

18 Moravcsik: What Can We Learn from the Collapse of the European Constitutional Project?, S. 237.

19 Simon Hix: Why the EU needs left-right politics, in: Politics: the Right or the Wrong Sort of Medicine for the EU?, Notre Europe Policy Paper Nr. 19/2006, abrufbar unter: http://www.notre-europe.asso.fr/IMG/pdf/ Policypaper19-en.pdf (letzter Zugriff: 10.10.2006), S. 7.

20 Larry Siedentop: A crisis of legitimacy, in: Prospect 112, Juli 2005, S. 26.

21 Hix: Why the EU needs left-right politics, S. 7.

22 Andreas Follesdal and Simon Hix: Why there is a democratic deficit in the EU: a response to Majone and Moravcsik, European Governance Papers Nr. C05 02/ 2005, abrufbar unter: http://www.connex-network.org/eurogov/pdf/egp-connex-C-05-02.pdf (letzter Zugriff: 10.10.2006), S. 25.

23 Loukas Tsoukalis: The JCMS Lecture: Managing Diversity and Change in the European Union, in: Journal of Common Market Studies 1/2006, S. 1-15, hier S. 6. 
economic and monetary policies be more orthodox or Keynesian? Should the EU have a more liberal or more restricted immigration policy?“24

Folglich ist eine größere Politisierung der Politikgestaltung unvermeidlich. Denen zufolge, die glauben, dass die EU ein demokratisches politisches System sein solle, ist dies jedoch auch unerlässlich, um die Legitimität des Systems in der Bevölkerung zu stärken. Eine Politisierung hätte etliche der in dieser Hinsicht wünschenswerte Auswirkungen: Erstens, weil sie die politischen Entscheidungsträger verantwortlich machen würde für ihren politischen Output. Der politische Schlagabtausch versetzt die Bürgerinnen und Bürger in Demokratien in die Lage, konkurrierende politische Programme und konkurrierende Kandidaten für öffentliche Ämter gegeneinander abzuwägen und diejenigen zu verdrängen, die ihre Macht nicht wie im Wählerauftrag vorgesehen gebrauchen. Zweitens, weil dieser Prozess der Deliberation die Politik dazu bringen würde, stärker auf sich verändernde Präferenzen einzugehen. Durch Deliberation bildeten und veränderten Bürgerinnen und Bürger ihre Meinungen über politische Wahlmöglichkeiten. ${ }^{25}$

Follesdal und Hix bestreiten die Behauptung, dass die EU sich mit Fragen befasse, die nicht wichtig für die Bürgerinnen und Bürger seien, indem sie darauf hinweisen, dass politische Fragen und Probleme dem politischen Prozess eigentlich endogen seien. Das heißt, dass bestimmte Fragen als nicht wichtig wahrgenommen werden, weil sie nicht politisiert wurden - und nicht andersherum. Es folgt, dass ,,apathy is likely to change if media and political parties start to claim that EU decisions impact on high-salience issues such as health care provision, education law and order, pensions and social security." 26 Darüber hinaus stimmen diese Autoren nicht überein mit Moravcsiks Argument, dass ein depolitisiertes EUSystem Output produziere, der den Präferenzen der Wählerinnen und Wähler entspreche. Ohne politischen Schlagabtausch gebe es keine Möglichkeit, sicher zu stellen, dass die Art der , aufgeklärten Bürokratie', die von Majone und Moravcsik bevorzugt wird, weiterhin auf sich verändernde Präferenzen eingehen wird - oder dass der nächste politische Führer ebenso wohlwollend sein wird. ${ }^{27}$

Wie vorab dargelegt argumentiert Moravcsik, dass diese Beschäftigung mit demokratischer Partizipation von einer Fixierung auf eine ,ideale Vorstellung von Demokratie“ herrühre, die in Bezug auf den Nationalstaat konzipiert wurde. In seiner Erwiderung entgegnet Hix, dass eine zunehmende Partizipation nicht nur im Hinblick auf theoretische, sondern auch auf pragmatische Effizienzgründe zwingend erforderlich sei. Eine Politisierung würde die Partizipation nicht auf Kosten der Effizienz verstärken, sondern vielmehr beide Dimensionen steigern: „The choice is not between reforming the EU to promote political leadership and policy efficiency or reforming the EU to promote more politics and democracy. In reality, one cannot exist without the other." 28

Hix weist darauf hin, dass das von Moravcsik beschriebene, hochgradig auf Konsens angelegte System der Gewaltenteilung und -verschränkung nicht nur radikalen Politikwandel verhindere - sondern generell die Annahme jeder politischen Entscheidung erschwere. Wenn Politiken einmal vereinbart seien, führten die hohen Stimmenanteile, die erforderlich sind, um eine Entscheidung rückgängig zu machen, dazu, dass Politiken, eingeschlossen “ würden, so dass sie nicht leicht an sich verändernde Präferenzen oder Zusammenhänge angepasst werden können. Sehr viel wissenschaftliche Aufmerksamkeit ist auf die Frage ge-

24 Hix: Why the EU needs left-right politics, S. 7.

25 Hix: Why the EU needs left-right politics.

26 Follesdal and Hix: Why there is a democratic deficit in the EU, S. 25.

27 Follesdal and Hix: Why there is a democratic deficit in the EU.

28 Hix: Why the EU needs left-right politics, S. 4. 
richtet worden, wie man das Potenzial für einen völligen institutionellen Stillstand in einer EU von 25 Mitgliedstaaten überwinden könne - der Verfassungsvertrag war natürlich ein Versuch, dieses Problem zu bewältigen. Hix stellt fest, dass aufgrund der noch fehlenden Einigung über eine Vertragsänderung die Politisierung ein effektives Mittel zur Stärkung der Handlungsfähigkeit der EU darstelle, während das bestehende System der Gewaltenteilung und -verschränkung radikalen Wandel verhindern und damit potenzielle Risiken minimieren würde. ${ }^{29}$

Des Weiteren diente das Scheitern der Ratifizierung für Hix dazu, die These hervorzuheben, dass ohne ein größeres Engagement der Bürgerinnen und Bürger in der EU die Stabilität des Systems in Gefahr sei. Im derzeitigen System haben diejenigen, die der Richtung von EU-Politik kritisch gegenüber stehen, keine Wahl, als sich gegen die europäische Integration insgesamt zu stellen, da sie keine Möglichkeit haben, den Entscheidungsprozess zu beeinflussen. Viele Wissenschaftlerinnen und Wissenschaftler haben zum Beispiel angemerkt, dass dies in den Debatten um den Verfassungsvertrag beobachtet werden konnte, die stärker auf die Orientierung der politischen Ökonomie der EU fokussiert waren als auf den Inhalt des Dokumentes selber: „In the debate on the Constitutional Treaty in several member states it became clear that much opposition to the treaty was founded on the belief that if the treaty were accepted it would hasten the dismantling of the European social model, and the turn to neo-liberal policies throughout Europe. However, since many of the neo-liberal elements to which the objections were strongest were already contained in the existing treaties, they were unaffected by the rejection of the Constitutional Treaty. "30 Politisierung würde dieses Problem durch die Bereitstellung institutioneller Mechanismen überwinden, um , debate and contestation about politics in, not only of, the EU“'31 zu erzeugen. Im Ergebnis würde die Effizienz der EU als System nicht länger durch einen Mangel an Unterstützung seitens der Bevölkerung behindert werden.

Für Hix stellt das Scheitern des Ratifikationsprozesses für die EU keine Katastrophe dar, weil erstens der Verfassungsvertrag in jedem Fall nicht weit genug ging bei der Umsetzung der Art von Reformen, die für die Bewältigung dieser Probleme notwendig waren, und zweitens, weil die notwendigen Reformen innerhalb der Grenzen der bestehenden Verträge erreicht werden können. Der wachsende Korpus von Arbeiten über politische Auseinandersetzung, in dem Hix selbst führend ist, hat beträchtliche empirische Belege dafür geliefert, dass die Politik innerhalb der europäischen Institutionen zunehmend von einer ideologischen Konfliktlinie bestimmt wird, die entlang der traditionellen territorialen Konfliktlinien verläuft. ${ }^{32}$ Was Hix problematisch findet, ist, dass diese Konfliktlinien völlig von den Wählerpräferenzen abgekoppelt sind: „European citizens have very little information about the emerging politics inside the EU institutions and so cannot identify the protagonists and the positions they represent. "33 Die Wahlen zum Europäischen Parlament seien nicht an politi-

29 Hix: Why the EU needs left-right politics.

30 Andrew Gamble: The European Disunion, in: British Journal of Politics and International Relations 1/2006, S. 34-49, hier S. 46.

31 Follesdal and Hix: Why there is a democratic deficit in the EU, S. 30.

32 Simon Hix: Legislative Behaviour and Party Competition in the European Parliament: an Application of Nominate to the EU, in: Journal of Common Market Studies 4/2001, S. 663-688; Simon Hix/Amie Kreppel: From Grand Coalition to Left-Right Confrontation: Explaining the Shifting Structure of Party Competition in the European Parliament, in: Comparative Political Studies 1/2 /2003, S. 75-96.; Christina Zimmer/Gerald Schneider/ Michael Dobbins: The Contested Council: Conflict Dimensions of an Intergovernmental EU Institution, in: Political Studies 2/2005, S. 403-422.

33 Hix: Why the EU needs left-right politics, S. 23. 
sches Verhalten gekoppelt, und daher sei es für Wähler unmöglich, die politisch Verantwortlichen zu bestrafen oder zu belohnen.

Im Lichte der Verfassungskrise führt Hix seine Beschreibung der EU als ein politisches System, das direkt mit anderen politischen Systemen verglichen werden kann, näher aus. Ein solcher Vergleich zeigt jedoch, dass die EU im Hinblick auf ihren demokratischen Input zurück bleibt - was wiederum die Effizienz und Legitimität ihres Outputs bedroht. Sein ,Re$z e p t^{\star}$ für die EU ist eine, graduelle' Reformagenda, um diese Probleme zu beseitigen. Die notwendigen Reformen würden eher politischen Willen als eine Vertragsänderung erfordern und politischen Wettbewerb zwischen konkurrierenden Kandidaten und Programmen für das Amt des Kommissionspräsidenten, größere Transparenz im Ministerrat sowie eine Steigerung der Gestaltungsmacht der Mehrheitsfraktion im Parlament hinsichtlich der europapolitischen Agenda einschließen. ${ }^{34}$ Das Scheitern des Verfassungsvertrages habe gezeigt, dass die EU eine kritische Phase in ihrer Entwicklung erreicht habe. Seine Prognose ist, dass es künftig einen höheren Grad an Politisierung geben wird, da Entscheidungen darüber getroffen werden, wie das etablierte System zu gestalten sei. Wenn gegen diese Entwicklung jedoch Widerstand geleistet würde, dann würden die Verlierer dieser politischen Entscheidungen möglicherweise die zukünftige Stabilität des Systems bedrohen.

\section{,Management von Vielfalt‘: Integration durch Institutionalisierung}

Eine dritte Lesart des Scheiterns der Ratifizierung sieht in der EU ein beispielloses supranationales Experiment für das Management von Vielfalt. Damit sei sie eher für organische, stückweise Prozesse als für ,große Entwürfe ' geeignet, die sie in die Strukturen, Prozeduren und das Vokabular einengen, die entweder in Bezug auf Staaten oder auf internationale Regime konzipiert worden sind.

Olsen zum Beispiel geht von der Annahme aus, dass selbst wenn bewusste institutionelle Reformen eine Rolle in der Formung des politischen Systems spielen, diese nicht die bedeutendsten Mechanismen des Wandels in einer komplexen und dynamischen politischen Ordnung wie der EU seien. Olsen merkt an, dass der Konstitutionalisierungsprozess in der Sprache einer institutionellen Reform von oben formuliert wurde. Dieser rhetorische Gestus ,used an institutional engineering language, arguing what would be the best institutional tools for common purposes and how the union could be made more effective." 35 Ziel sei es gewesen, eine Balance zwischen den verschiedenen Spannungen zu erreichen, die im Herzen eines jeden politischen Systems bestehen - zum Beispiel zwischen der Autonomie seiner konstitutiven Einheiten einerseits und effektiver Koordinierung andererseits sowie zwischen Effizienz und Partizipation. In anderen Worten war der Verfassungsvertrag somit ein bewusster Versuch, das Verhältnis zwischen Einheit und Vielfalt zu organisieren. ${ }^{36}$

Olsen hebt jedoch hervor, dass eine Reihe von Kernbedingungen erfüllt sein müssen, wenn eine solche institutionelle Konstruktion Erfolg haben soll: Es muss auf der Basis von stabilen Präferenzen und einer gut gestalteten Strategie für ihre Verwirklichung eine klare, einvernehmliche Vorstellung davon geben, was die Reformen zu erreichen bezwecken. Die Reformer müssen ein Mandat sowie die Macht und Autorität für die Durchführung der Reformen haben. ${ }^{37}$

34 Hix: Why the EU needs left-right politics, S. 23.

35 Johan P. Olsen: The Political Organization of Europe: Differentiation and Unification, Arena Working Paper 23/2005, S. 20.

36 Olsen: The Political Organization of Europe, S. 20.

37 Johan P. Olsen: Reforming European Institutions of Governance, in: Journal of Common Market Studies 4/ 2002, S. 581-602. 
Alle diese Bedingungen fehlten auf der EU-Ebene. Erstens würden Reformer in ihrem Versuch behindert, die Spannungen zwischen den widerstreitenden politischen Integrationsprojekten, die Gegenstand dieses Aufsatzes sind, miteinander in Einklang zu bringen. „Institutional engineers cannot assume a shared vision of the future Europe, providing clear normative criteria of institutional improvement. "38 Da jede unterschiedliche Konzeption der EU eine andere Beziehung zwischen Effizienz und Partizipation sowie zwischen Entscheidungsträgern und ihren Entscheidungsunterworfenen vorsieht, gibt es weder eine offensichtliche Wählerschaft, die ein Mandat erteilen könnte, noch ein abgestimmtes Verfahren, um es bereit zu stellen. Sollten Reformen durch ein Referendum beschlossen werden? Oder durch die Regierungen? Vom europäischen Volk, oder von den Völkern? Darüber hinaus seien die Präferenzen und Identitäten der Akteure eher veränderlich als stabil, da Integration noch immer ein umstrittener und dynamischer politischer Prozess ist. Der EU mangele es für die Bewältigung einer umfassenden Reform daher an einem gemeinsamen Vokabular, gemeinsamen Konzepten und einem kognitiven Rahmen. ${ }^{39}$ Schließlich gebe es, da die Verteilung von Autorität und Macht offen ist ${ }^{40}$, kein einzelnes Machtzentrum, das die politische Ordnung maßgeblich verändern könne.

Die Debatten während des Konvents zeigten deutlich, dass die vielfachen Spannungen bestehen bleiben - ob sie nun territoriale Fragen oder Einstellungen zu Integration betreffen. ${ }^{41}$ Angesichts dieser Heterogenität sei es für den Verfassungsvertrag unmöglich gewesen, die unterschiedlichen politischen Integrationsprojekte miteinander in Einklang zu bringen und die Frage nach der Natur der politischen Ordnung der Union zu klären. Viele Kommentatoren haben nahe gelegt, dass die resultierende Ambivalenz im Herzen des Dokumentes der Grund für seinen Untergang war: ,There had always been tensions between two different bases of EU legitimacy - one deriving from treaties between states and the other deriving from democracy and citizenship - but never before had the collision between them been so serious. The Constitutional Treaty was based on a fatal, and perhaps unavoidable, ambiguity between the two and ended up pleasing almost no one. "42

Was aber sind dann die Implikationen des Misserfolges, sich nicht über die Natur der politischen Ordnung der EU geeinigt zu haben, für ihre, finalité politique'? Kündigt dies in einer Union von 25 Mitgliedstaaten eine unvermeidliche Desintegration an? Laut Olsen ist das genaue Gegenteil möglich. Er erinnert uns daran, dass - während es keine bewusste Vertragsreform geschafft oder auch nur den Versuch unternommen habe, das Problem der konkurrierenden politischen Integrationsprojekte zu lösen - ,the European Union has shown ability to live with an open ended process and enduring tensions and inconsistencies, not only in terms of policies but also institutional arrangements. “43 In der Tat ist es wahrscheinlich, dass diese konzeptionelle Offenheit den Schlüssel für das Verständnis dafür geliefert hat, wie die EU es so lange bewerkstelligt hat, zunehmende Vielfalt mit der Vertiefung ihres Programms und ihrer institutionellen Kapazität unter einen Hut zu bringen.

Institutionalisten wie Olsen warnen davor, das Ausmaß der EU-Integration rein anhand formaler und rechtlicher Maßeinheiten zu analysieren. Um die Tiefe der Integration und die Natur der Mechanismen des Wandels zu erfassen, müssten Wissenschaftlerinnen und Wissenschaftler den Akzent nicht auf ,institutionenfreie' Modelle des intergouvernementalen

38 Olsen: Reforming European Institutions of Governance, S. 588.

39 Olsen: Reforming European Institutions of Governance, S. 594.

40 Olsen: The Political Organization of Europe.

41 Olsen: The Political Organization of Europe.

42 Gamble: The European Disunion, S. 35.

43 Olsen: The Political Organization of Europe, S. 18. 
Bargainings setzen, sondern darauf, wie die Institutionen, die mit den Verträgen errichtet wurden, mit der Zeit in Praktiken und Verhaltensweisen umgesetzt würden. Da kein Konsens darüber erzielt worden sei, wie Regieren auf der supranationalen Ebene konfiguriert und legitimiert werden sollte, seien die Spannungen im Herzen des Systems pragmatisch und fallweise gelöst worden. Unterschiedliche institutionelle Arrangements und Verfahren hätten sich entwickelt, um die Anforderungen der verschiedenen Politikbereiche zu erfüllen. Innerhalb eines jeden dieser institutionellen Netzwerke hätten sich die Akteure durch tägliche Praxis, Lernen, Verhandlung und Problemlösung an neue Anforderungen angepasst. ${ }^{44}$ Diese Lern- und Anpassungsprozesse hätten zu graduellen Veränderungen in den Wahrnehmungsmustern geführt. ${ }^{45}$ Schrittweise Maßnahmen hätten einen umgestaltenden Wandel bewirkt, der stufenweise fest verwurzelt sei und das Verhalten der institutionellen Akteure im Ablauf der Zeit eingeschränkt und beeinflusst habe. ${ }^{46}$

Aus dieser Perspektive ist die beste Beschreibung der EU die, die sie weder als eine Problemlösungsgemeinschaft sieht, deren Legitimität indirekt durch ihre Mitgliedstaaten bereitgestellt wird, noch als eine demokratische politische Ordnung, die mit einem Nationalstaat vergleichbar wäre, sondern die sie als einen dynamischen Prozesses versteht, der auf das Managen von Vielfältigkeit abzielt und dessen Konturen durch die Praxis definiert werden. Integration hat nicht in einer einheitlichen und gleichförmigen Art quer durch unterschiedliche Politikbereiche stattgefunden. Stattdessen ist sie in funktionaler, territorialer und temporaler Hinsicht differenziert gewesen. Die daraus entstandene ungleichmäßige Institutionalisierung hat die Herausbildung eines Zentrums unterdrückt und eine heterogene politische Ordnung mit mehreren Ebenen und Zentren erzeugt. ${ }^{47}$

Olsens Rezept für die EU ist, dass ein Konstitutionalisierungsprozess in solch einer verschiedenartigen politischen Ordnung sowohl kontraproduktiv als auch unnötig ist. Die Fähigkeit, täglich zu lernen und sich anzupassen, die innerhalb der Institutionen des Regierens stattfindet, decke, wenn sie gut entwickelt sei, den Bedarf an einem großen konstitutionellen Design und einer Reform, was in einer Situation der strittigen Zielvorstellungen hinsichtlich der politischen Ordnung aller Wahrscheinlichkeit nach Krisen hervorrufen würde. ${ }^{48}$

Hieraus folgt, dass Olsens Vorhersage für den zukünftigen Entwicklungspfad der EU trotz der Herausforderung, eine gesteigerte Vielfalt miteinander in Einklang zu bringen, optimistisch ist. Die institutionalistische Perspektive behauptet nicht, dass die EU einen Gleichgewichtszustand erreicht habe. Sie hält sowohl eine neuerliche Erweiterung als auch Vertiefung für möglich, warnt aber, dass dieser Entwicklungspfad nicht von oben vorherbestimmt oder festgelegt werden kann. Der wichtigste Faktor, der eine zukünftige Erweiterung und Vertiefung ermögliche, wäre ein flexibler Ansatz anstelle eines ,one size fits all'-Integrationsansatzes, der das politische System in die Lage versetze, sich schrittweise und bedarfsgemäß zu entwickeln: „Uneven and flexible integration, the principle that some member states can cooperate more closely than others, opt-out arrangements, the pillar system, the principles of subsidiarity and proportionality, the many and complex procedures in use, and the different time-scripts and rhythms of change, can all be seen as ways of balancing unity and diversity in a polity with limited community and shared purpose.“49

44 Olsen: The Political Organization of Europe.

45 Beate Kohler-Koch: The transformation of governance in the European Union, London 1999.

46 Kenneth Armstrong/Simon Bulmer: The Governance of the Single Market, Manchester 1998.

47 Olsen: The Political Organization of Europe, S. 17.

48 Olsen: The Political Organization of Europe, S. 23.

49 Olsen: The Political Organization of Europe, S. 19. 


\section{Kritik aus der Perspektive des Systembildungs-Ansatzes}

Während die Vielfalt der methodologischen Ansätze in der EU-Forschung zu begrüßen ist, haben Wessels und andere überzeugend argumentiert, dass weiterhin Versuche unternommen werden müssen, eine allgemeine Theorie zu ermitteln, die nicht nur auf die statische Meso-Ebene blickt, sondern auch hilft, die Gestalt und Energie der Integrationsdynamiken zu verstehen. ${ }^{50}$ Ein kürzlich unternommener Versuch, einen solchen ,holistischen * Ansatz für den Integrationsprozess zu entwickeln, hat sich aus der Systembildungstheorie der modernen Staatsbildung entwickelt, der Stein Rokkan den Weg gebahnt hat. Bartolini hat versucht, die von Stein Rokkan entwickelten Konzepte und Werkzeuge auf die Analyse des europäischen Integrationsprozesses anzuwenden. Wie Rokkan konzentriert sich auch Bartolini auf die ,longue durée " und stellt die Schaffung der Europäischen Union in den breiteren Rahmen der Entwicklung Europas vom Römischen Reich bis zur Bildung der modernen Staaten. ${ }^{51}$ Bartolini beschreibt europäische Integration aus dieser Perspektive als ,,a new phase of nation-state boundary transcendence resulting in the de-differentiation of European polities after five centuries of a progressive differentiation" ${ }^{52}$ Der Auslöser für diesen Prozess sei die Schwächung des Staatensystems im Kontext der Globalisierung der Finanzmärkte im 20. Jahrhundert gewesen.

Dieser Integrationsentwurf stellt die Darstellung der EU als eine reine „Problemlösungs-

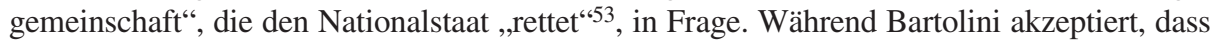
die EU eher eine Antwort auf statt die Ursache der nationalstaatlichen Unzulänglichkeit angesichts des internationalen Kapitalismus darstellt, betrachtet er Integration trotzdem als eine direkte Herausforderung der politischen Autorität des Nationalstaates. ${ }^{54}$ Der Kontrollverzicht über rechtlich-administrative und ökonomische Grenzen habe Folgen sowohl für die Systemgestaltung (Loyalitäten und Solidarität) und die interne politische Strukturierung (Konfliktlinien und Kanäle der Repräsentation) auf der nationalen Ebene. Wenn gesellschaftliche Akteure die nationale Arena verließen, um ein Mitspracherecht im neuen Zentrum auszuüben, wirke sich dies auf die staatliche Kontrolle über Umverteilung und kulturelle Symbole aus, von denen demokratische Prozesse in Staaten und Wohlfahrtssystemen abhängen. Bartolini sieht daher die von Moravcsik und Majone beschriebene klare Trennung der Zuständigkeiten zwischen der nationalstaatlichen und der EU-Ebene, bei der die Mitgliedstaaten vollständige Kontrolle über die Regierungstätigkeit behalten, als einen Trugschluss: „What cannot be maintained is the thesis of a division of labour between the EU and the nation state according to which the EU should be exclusively responsible for the regulation/deregulation of the market (in a way that is as independent as possible from political pressures) while the nation state should retain responsibility for introducing the necessary compensatory measures." 55

Bartolini teilt die Ansicht von Hix, dass das Management des Binnenmarktes fundamentale politische Konsequenzen habe. Sowohl positive als auch negative Formen von Integration würden unvermeidlich nationale Mechanismen der politischen Repräsentation und Legitimation untergraben, was $\mathrm{zu}$ einer nationalen politischen Destrukturierung führe. Bartolini findet jedoch das Rezept von Hix, diesen Zustand durch die Konstruktion eines

50 Wolfgang Wessels: Cleavages, controversies and convergence in European Union Studies, in: Michelle Cini and Angela Bourne: European Union Studies, Basingstoke 2006, S. 233-246, hier S. 238.

51 Stefano Bartolini: Restructuring Europe: Centre Formation, System Building, and Political Structuring Between the Nation State and the European Union, Oxford 2005, S. xii.

52 Bartolini: Restructuring Europe, S. 375.

53 Alan Milward: The European rescue of the Nation-State, London 1992.

54 Bartolini: Restructuring Europe, S. 368.

55 Bartolini: Restructuring Europe, S. 407. 
größeren Maßes an Politisierung auf der europäischen Ebene zu beheben, hoch problematisch.

Sein erster Einwand ist, dass Politisierung nicht auf Fragen beschränkt bliebe, die analog zu nationalen Konfliktlinien sind, was es der europäischen Politik erlauben würde, entlang denselben Linien strukturiert zu sein wie nationale Angelegenheiten. Es gebe wenig Chancen, dass eine neue, europäische Struktur politischer Konfliktlinien geschaffen werden könnte, die der nationalen gleicht, denn die Einstellungen zum Integrationsprozess seien nur schwach an parteistrategische Orientierungen gekoppelt und gingen quer durch Interessengruppen und politische Organisationen, die ansonsten geschlossen seien. Im Ergebnis führe dies dazu, dass „European coordination of groups that are isomorphic to national decisions rest on very thin support. "56 Stattdessen behauptet Bartolini, dass sich politische Kämpfe sehr viel wahrscheinlicher auf konstitutionelle Fragen konzentrieren würden, das heißt auf solche Fragen, die Mitgliedschaft, Kompetenzen und Entscheidungsverfahren betreffen und von denen er im Gegensatz sowohl zu Moravcsik als auch zu Hix nicht glaubt, dass sie bereits gelöst seien. ${ }^{57}$ Welchen Grund gibt es dafür zu glauben, dass der Streit lediglich Rechts-Links-Themen betreffen wird, fragt er, wenn die Bereiche, die die europäischen Bürgerinnen und Bürger am meisten beschäftigen, sich auf Konflikte über die Reichweite der EU, ihre Kompetenzen und sogar ihre Existenz beziehen?

Zweitens ist Bartolini sehr skeptisch angesichts des Optimismus von Hix hinsichtlich des Potenzials für europäische Parteien, als Verbindungskanäle zwischen den Präferenzen der Wählerinnen und Wähler und dem politischem Verhalten innerhalb der europäischen Institutionen zu agieren. Er schreibt ihre Geschlossenheit bei Abstimmungen einem Mangel an Parteidisziplin, einer geringen Sichtbarkeit und den Wahlkosten zu anstatt einem wirklichen ideologischen Zusammenhalt. Dies führt ihn zu der Schlussfolgerung, dass Politisierung tatsächlich den internen Zusammenhalt in den Parteigruppierungen auflösen würde, anstatt ihn zu stärken. ${ }^{58}$

Sein dritter Einwand ist der, dass Politisierung unvermeidlich unrealistische Erwartungen darüber erzeugen würde, was tatsächlich erreicht werden könnte innerhalb der bestehenden, festgelegten Ziele der EU. Angesichts der Tatsache, dass es keine klare Gewaltentrennung gebe und dass die Kompetenzen der Union in Bezug auf eine Liste von substanziellen - und lose definierten - Zielen festgehalten seien, bezweifelt Bartolini, dass in der EU tatsächlich ein konstitutionelles Abkommen getroffen worden sei. Die Besonderheit dieses Arrangements sei, dass die Verträge tatsächlich die Kompetenzen davor schützten, in einer politischen Auseinandersetzung entschieden $\mathrm{zu}$ werden, anstatt ,the procedures for selecting those who are allowed to take decisions and the procedures for taking those decisions. "59 einfach schriftlich zu fixieren. Man könne daher von einer ,Konstitutionalisierung ' der wirtschaftlichen Rechte und der marktschaffenden Ziele sprechen, nicht aber von einer Konstitutionalisierung der entsprechenden politischen oder sozialen Rechte ${ }^{60}$ Folglich sei die Förderung einer öffentlichen Deliberation über alternative politische Mandate zum Scheitern verurteilt. Bartolini sieht das Beispiel eines politischen Mandates als eines an, das eine sich ausweitende Geldpolitik, europäische soziale Mindeststandards, aktive Beschäftigungs-

56 Bartolini: Restructuring Europe, S. 394.

57 Stefano Bartolini: Should the Union be politicised? Prospects and Risks, in: Politics: The right or the wrong sort of medicine for the EU?, Notre Europe Policy Paper Nr. 19/ 2006, abrufbar unter: http://www.notre-europe.asso.fr/IMG/pdf/Policypaper19-en.pdf (letzter Zugriff: 10.10.2006).

58 Bartolini: Should the Union be politicised?

59 Bartolini: Restructuring Europe, S. 410.

60 Bartolini: Restructuring Europe, S. 410. 
oder Fiskalpolitik oder einen radikalen Wandel in der Agrarpolitik mit sich bringen würde. Wenn ein solches Mandat Rückhalt in der Bevölkerung finden würde, könnte es dann dazu beitragen, institutionellen Stillstand zu überwinden, wie Hix behauptet? „The answer is NO. Such a political mandate would be frustrated by the autonomy of the European Central Bank, by the case law of the European Court of Justice, by the blocking vetoes in the Council.“61

Bartolini argumentiert daher, dass das institutionelle Arrangement der Union gegen die Entstehung politischer Strukturierungsmechanismen auf der erweiterten territorialen Ebene spricht, um die politische Destrukturierung auf der nationalen Ebene zu kompensieren. Dieses institutionelle Arrangement einfach von oben abzuändern sei jedoch keine plausible Lösung - vielmehr könnte dieses Rezept schlimmer sein als die Krankheit: „Any institutional democratization without political structuring would be potentially catastrophic. "62 Bartolinis theoretischer Rahmen hält daran fest, dass politische Strukturierung von der Systembildungs-Kapazität einer territorialen Ordnung abhänge, das heißt von der Entwicklung eines gewissen Ausmaßes an kultureller Identifikation und Solidarität zwischen den Bürgerinnen und Bürgern dieser Ordnung und in Richtung auf die Institutionen des neuen Zentrums. Dies setze nicht die Erfordernis eines ethnokulturellen Demos voraus, da eine politische Gemeinschaft auf festgelegten Kodexen staatsbürgerlicher Pflichten, politischen Rechten oder einer Form des „republikanischen Patriotismus“ aufgebaut werden könne. Entscheidend sei jedoch, dass diese Art von interner Strukturierung nur sehr schwer, wenn nicht gar überhaupt nicht stattfinden könne, wenn territoriale oder Mitgliedschafts-Grenzen, die in der Europäischen Union offen bleiben, fehlten. ${ }^{63}$

Während Bartolini daher die Ansicht Olsens - der auch mit den theoretischen Werkzeugen der politischen Systembildung arbeitet - teilt, dass die EU eine sich ständig verändernde und lose begrenzte Ordnung ist, findet er diesen Zustand grundlegend problematisch. Die Theorie der politischen Entwicklung, auf der er aufbaut, behauptet, dass ,,some degree of coherence is necessary between cultural, economic, coercion and politico-administrative boundaries of any modern political formation." ${ }^{34}$ Wenn es einer politischen Ordnung an geschlossenen Grenzen mangele und es nur wenige Barrieren gebe, um sie zu verlassen, sei die Wahrscheinlichkeit, dass eine politische Strukturierung auftritt, niedrig. Da Akteure nicht , eingeschlossen“ seien in die Politikgestaltung und Loyalitäten könne sich kein Vertrauen entwickeln, und die ,organisatorische Hierarchie ‘ werde sich nur geringem Druck ausgesetzt sehen, Mechanismen zu entwickeln, um auf eine Mitsprache durch die Bürgerinnen und Bürger zu reagieren. ${ }^{65}$ Der funktional, territorial und zeitlich differenzierte Prozess der europäischen Integration, den Olsen als den Schlüssel zum Management von Vielfalt feiert, habe nationalstaatliche Grenzen aufgelöst, aber habe diese nicht auf der EU-Ebene wieder miteinander verbunden. Die daraus resultierende , unterschiedliche Grenztranszendenz ' habe zu einer Fragmentierung der Orte der Macht und der Entscheidungsfindung geführt und die Schaffung eines stabilen maßgebenden Zentrums, welches es politischen Kräften zu gedeihen ermöglicht, verhindert. So lange die EU fortfährt, sich stückweise auf der Basis von verfassungsmäßig geschützten wirtschaftlichen Zielen und Rechten, aber ohne eine Vereinbarung über die grundlegenden politischen Dilemmata und Spannungen zu entwickeln, solange könne sich keine stabile politische Ordnung entwickeln: „The scattered elements of

61 Bartolini: Should the Union be politicised?, S.39.

62 Bartolini: Restructuring Europe, S. 408.

63 Bartolini: Restructuring Europe.

64 Bartolini: Restructuring Europe, S. 410

65 Bartolini: Should the Union be politicised?, S. 47. 
identities, interests and institutions need to be reconciled in some way into a new coherent order. If this reconstruction does not occur at some level other than the nation state, then tensions, conflicts and problems are likely to emerge that could jeopardise the features specific to European civilisation." 66

\section{Schlussfolgerungen}

Vielleicht der einzige Bereich, in dem sich ein Konsens zwischen allen vier untersuchten theoretischen Perspektiven abzeichnet, ist der, dass eine große Diskrepanz zwischen der Rhetorik, die den Verfassungsvertrag umgab, und seinem realen Inhalt bestand. Vieles von diesem Diskurs implizierte, dass es das Ziel des Konstitutionalisierungsprozesses sei, fundamentale politische Fragen und Spannungen in Bezug auf die Natur der politischen Ordnung der EU zu klären und zu lösen. Doch die Reformer waren pragmatisch genug, um zu erkennen, dass, wenn sie auch nur versucht hätten, Antworten auf diese Fragen zu geben, ein Entwurf des Dokumentes schlicht die widersprüchlichen Zielvorstellungen sichtbar gemacht hätten, die dem Prozess zu Grunde lagen, anstatt diese näher zusammen zu rücken. Die Tatsache, dass der Verfassungsvertrag keine, magischen“ Antworten auf die politischen Fragen im Herzen des Integrationsprozesses bot, trägt zu der Erklärung bei, warum die Mehrheit der in diesem Beitrag vorgestellten Autoren nicht glaubt, dass das Scheitern der Ratifizierung des Verfassungsvertrags die EU in einer schlechteren Lage zurücklässt als die, aus der sie gestartet war.

Die Implikationen dieser gegenwärtigen Lage der EU für ihren zukünftigen Entwicklungspfad hängen davon ab, ob wir glauben, dass das System als ein unbegrenzter Prozess mit einem offenen Ende gut funktioniert, oder ob wir das Gefühl haben, dass ein gewisses $\mathrm{Ma} ß$ an Einvernehmen über seine politische, territoriale und funktionale Finalität erforderlich ist. Sie hängen darüber hinaus davon ab, ob davon ausgegangen wird, dass die EU als politische Ordnung einen Gleichgewichtszustand erreicht hat, oder ob die Integration sich als ein dynamischer Prozess fortsetzt. Die in diesem Beitrag vorgestellten Autoren vertreten unterschiedliche Auffassungen hinsichtlich dieser Frage.

Moravcsik behauptet, dass die EU ihren Gleichgewichtszustand erreicht habe. Die logische Schlussfolgerung ist, dass es keine weitere Vertiefung geben wird, dass aber dieser Vertiefungsstopp wiederum die Voraussetzung schafft für eine mögliche weitere Erweiterung. Für Hix hat die EU ebenfalls einen Gleichgewichtszustand erreicht, aber diese Vorstellung eines ,Gleichgewichts‘ schließt eine weitere Vertiefung nicht aus. Da Hix sich eher mit der Funktionsweise der EU befasst als mit ihren dynamischen Entwicklungsprozessen, diskutiert er Erweiterung und Vertiefung nicht mit diesen Begrifflichkeiten. Die meisten Wissenschaftlerinnen und Wissenschaftler würden seine Vorstellung eines höheren Grades an Politisierung jedoch als eine Vertiefung des Integrationsprozesses verstehen, ungeachtet der daraus resultierenden Politiken. Im Gegensatz dazu geht Olsen davon aus, dass die EU keinen Gleichgewichtszustand erreicht hat, sondern noch immer ein dynamisches , politisches Gemeinwesen im Werden“ ist. Diese Dynamik werde sich fortsetzen; ihr sei aber am besten damit gedient, dass man den Zweck, die Struktur und die Ziele dieser politischen Ordnung flexibel hält.

Bartolinis Argumentation legt im Gegensatz dazu nahe, dass die Fortführung der Integrationsdynamiken, die bis jetzt als treibende Kraft hinter der Entwicklung der Union gestanden haben, höchst problematisch wäre. Eine weitere Vertiefung in Form einer Demokratisierung ohne eine Schließung der Grenzen würde sowohl die Union als auch die Basis des repräsen-

66 Bartolini: Restructuring Europe, S. 411. 
tativen Prinzips, die Wohlfahrtssysteme und die Solidarität in ihren Mitgliedstaaten gefährden. Eine Territorialisierung wäre eine notwendige Vorbedingung für eine Vertiefung, weil eine politische Strukturierung nur aus einer Kohärenz zwischen ökonomischen, kulturellen und politischen Grenzen entstehen kann. Eine weitere Erweiterung ohne klare geografische Abgrenzung würde daher eine weitere Vertiefung in Form einer politischen Strukturierung unmöglich machen.

Die unvermeidliche Frage, die diese Kritik aufwirft, ist, ob es wünschenswert oder tatsächlich möglich wäre, die geschlossenen Grenzen und die politische Strukturierung, die sich im Nationalstaatskontext entwickelt haben, einfach zu reproduzieren. Die EU wurde konzipiert als ein alternatives politisches System, um die Dinge umzusetzen, die in den Nationalstaaten nicht länger effektiv geleistet werden konnten. Ihr komplexes System von flieBenden und differenzierten Grenzen entwickelte sich als Antwort auf die Herausforderungen der Interdependenz in einer sich wandelnden internationalen Ordnung. Was wären dann Sinn und Zweck des Versuchs, die EU in eine vergrößerte territoriale Ordnung umzuwandeln, die ihren Mitgliedstaaten - abgesehen von der Größe - in jeder Hinsicht ähnelt?

Ein Fokus auf diese Fragen dient als Erinnerung daran, dass die Schwächung traditioneller Formen politischer Strukturierung innerhalb des größeren Bildes eines sozialen, politischen und ökonomischen Wandels gesehen werden muss. Gesellschaften sind heute nicht länger nach traditionellen Klassen- und Parteibindungen gegliedert. Die Zivilgesellschaft ist weniger geschlossen, und ihre Fügsamkeit sowie das Vertrauen in Autorität ist gebröckelt. Die politische Antwort auf das Scheitern der Ratifizierung des Verfassungsvertrages nähert sich einem Konsens darüber an, dass der gegenwärtige Fokus nicht auf der konstitutionellen Natur der Union liegen sollte, sondern darauf, wie man die Antworten geben kann, die die Menschen in Reaktion auf den skizzierten Wandel verlangen. ${ }^{67}$ Tony Blairs Rede vor dem Europäischen Parlament nach den negativen Referenden stellen das Scheitern des Ratifizierungsprozesses in diesen breiteren Kontext: „It is not a crisis of political institutions, it is a crisis of political leadership. People in Europe are posing hard questions to us. They worry about globalisation, job security, about pensions and living standards. “68

Die EU ist nicht die Ursache dieser Krise - sie ist eine Antwort auf sie. Sie ist nicht die Ursache der Transformation des Regierens, sondern ein Experiment in gelenkter Internationalisierung. Wissenschaftler haben eine Schlüsselrolle bei der Aufzeichnung und Auswertung des Entwicklungspfades dieser „experimentellen Union“69, indem sie Mechanismen des Wandels identifizieren und Strategien vorschlagen, um die Arbeitsweise ihrer Infrastruktur effektiver zu gestalten. Die Vielfalt der wissenschaftlichen Reaktionen auf die Frage, welche Art eines politischen Systems die Europäische Union darstellt, reflektiert die Vielzahl der widerstreitenden Zielvorstellungen, die unter Politikern und Bürgerinnen und Bürgern nebeneinander bestehen. Versuche, diese grundlegenden politischen Spannungen zu lösen, haben sich bislang weder als hilfreich noch als möglich erwiesen. Die Kernfrage für Wissenschaftler, Praktiker sowie die Bürgerinnen und Bürger ist, ob sich dieses System der ,gelenkten Internationalisierung“, das sich in Westeuropa nach dem Zweiten Weltkrieg

67 José Manuel Barroso: Uniting in Peace: the Role of Law in the European Union, Jean Monnet Vorlesung, Europäisches Hochschulinstitut Florenz, 31. März 2006; Institut für Europäische Politik (Hrsg.): EU-25 Watch Nr. 3, Juli 2006, Berlin, abrufbar unter: http://www.iep-berlin.de/publik/EU25-Watch/EU-25_Watch-No3.pdf (letzter Zugriff 10.10.2006).

68 Tony Blair: Speech to the European Parliament, 23 Juni 2005, abrufbar unter: http://www.fco.gov.uk/Files/ kfile/UKEUPresidency2005_Sp_PM_EuropeanParliament_230605,0.pdf (letzter Zugriff: 10.10.2006).

69 Brigid Laffan/Rory O`Donnell/Michael Smith: Europe’s Experimental Union: Rethinking Integration, London 1999. 
entwickelt hat, weiterhin im einem den gesamtem Kontinent umfassenden Maßstab an die intensiven und weit reichenden Prozesse der Globalisierung, des Wettbewerbsdrucks, der Sicherheit und der Nachhaltigkeit anpassen kann.

Übersetzung aus dem Englischen von Dr. Anne Faber.

Der Beitrag ist Teil des EU-CONSENT Projektes (www.eu-consent.net) einem europaweiten Forschernetzwerk zu den Auswirkungen von Vertiefung und Erweiterung der EU im Rahmen des 6. Forschungsrahmenprogramms der Europäischen Union.

\section{Deutsche Ratspräsidentschaft Aufruf zur Einreichung von Manuskripten}

Die deutsche EU-Ratspräsidentschaft im ersten Halbjahr 2007 ist ein Schwerpunkt der redaktionellen Planung für die vier Ausgaben der Zeitschrift integration des kommenden Jahres. Daher rufen wir dazu auf, Manuskripte aus den Disziplinen Politik-, Rechts-, Wirtschafts- und Geschichtswissenschaft zum Thema „EU-Ratspräsidentschaft und deutsche Europapolitik“ bei der Redaktion integration einzureichen.

\section{Aspekte und Themenvorschläge}

- Berliner Erklärung aus Anlass des Jubiläums 50 Jahre Römische Verträge

- Vergleich vorheriger deutscher Ratspräsidentschaften

- zur allgemeinen Rolle, Funktion und Entwicklung der „Ratspräsidentschaft“ in der EU

- Ratspräsidentschaft und Verfassungsdebatte: Roadmap, Ratifizierungsprozess

- Bilanz des Juni-Gipfels, spezifischer Politikfelder bzw. der Ratspräsidentschaft im breiteren Kontext

- Deutsche Ratspräsidentschaft im Kontext deutscher Europapolitik

- die Dreierpräsidentschaft Deutschland - Portugal - Slowenien

Diese Liste dient lediglich als Anregung. Die Redaktion ist offen für Beiträge zu weiteren Aspekten der Ratspräsidentschaft. Manuskripte können auch in englischer oder französischer Sprache eingereicht werden. Sie würden dann im Falle einer Veröffentlichung übersetzt.

\section{Redaktionsschluss der einzelnen Ausgaben}

- Heft 1/2007: 17. November 2006

- Heft 2/2007: 16. Februar 2007

- Heft 3/2007: 17. Mai 2007

- Heft 4/2007: 17. August 2007

\section{Gutachterverfahren}

Themenvorschläge können jederzeit schriftlich oder telefonisch an die Redaktion integration gerichtet werden. Der Veröffentlichung von Manuskripten ist ein Gutachterverfahren vorgeschaltet. Nähere Informationen zum Gutachterverfahren sowie einige Autorenhinweise stehen unter http://www.iep-berlin.de/publik/integration/autorenhinweise. htm zum Download zur Verfügung.

\section{Redaktion integration}

Institut für Europäische Politik, Bundesallee 22, 10717 Berlin

E-Mail: barbara.lippert@iep-berlin.de

Tel. (030) 889134-0, Fax (030) 889134-99 